\title{
Dirac Equation in the Background of the Nutku Helicoid Metric
}

\author{
T. Birkandan, M.Hortaçsu
}

Physics Department, Faculty of Sciences and Letters

ITU 34469 Maslak, Istanbul, Turkey

\begin{abstract}
We study the solutions of the Dirac equation in the background of the Nutku helicoid metric. This metric has curvature singularities, which necessitates imposing a boundary to exclude this point. We use the AtiyahPatodi-Singer non local spectral boundary conditions for both the four and the five dimensional manifolds.
\end{abstract}

key words: APS boundary conditions, solutions of Dirac equation

E-mail addresses: hortacsu@itu.edu.tr, birkandant@itu.edu.tr

PACS number: 04.62.+v 


\section{Introduction}

New interesting solutions for gravitational instantons exist in the literature. One of them is the helicoid solution of Nutku [1,2]. Since this solution has curvature singularities, it has not been studied extensively aside from three articles $[3,4,5]$. These three papers study the solutions of the Dirac equation using this solution as a background. Our work on these solutions can be extended by studying them in a manifold with a boundary. Taking a domain that excludes the origin and infinity for the radial variable cures the problems associated with the infinities of these solutions.

One of us (M.H.), with collaborators, had studied the related problems in the presence of an instanton and a meron in the past $[6,7]$. Similar problems have been studied recently for a spherical bag [8 ]. In these three papers, to conserve self adjointness, as well as chirality and charge conjugation, spectral boundary conditions $[9,10]$ were used. The spectral boundary conditions were also extended to the Dirac equation with torsion $[11]$.

Here we study the solutions to the Dirac equation in the background of the Nutku helicoid metric. We use Euclidean signature for our metric. This makes our differential operator of the elliptic type. We find that although some of the solutions diverge at the origin, they are normalizable when integrated with the measure $d \tau \sqrt{g}$, where $\tau$ is the volume. We also see that the solution diverges as $x$ goes to infinity, since they are expressed in terms of modified Mathieu functions. Forcing them to be regular at the origin makes them divergent at infinity. Furthermore, the metric used has curvature singularities at the origin. Although one can define the index on certain noncompact manifolds, the conditions of "bounded geometry" are needed $[12,13]$. These are the conditions we do not meet. Thus we have to restrict our space at a boundary. This necessitates specifying the boundary conditions.

We know that local boundary conditions can be used in even dimensions, although, it was shown [6 ] that the spectral boundary conditions $[9,10]$ are the only self adjoint one that also conserves the $\gamma^{5}$ and the charge conjugation symmetry of the Dirac operator. The chiral Dirac operator, however, requires spectral boundary conditions in even dimensional spaces [14]. The non-local, spectral boundary conditions should be used for the odd dimensional case due to obstructions that were first pointed out by Atiyah and Bott [15 ]. Here we will study the problem both in odd and even dimensions. We will use the spectral boundary conditions in both cases.

The non-local spectral boundary conditions were introduced by Atiyah-Patodi-Singer while they were investigating the Hirzebruch signature theorem for manifolds with boundaries $[15,16,17]$. When a manifold has a boundary, we may use local elliptic boundary conditions, like those of Dirichlet, Neumann or Robin, if the manifold has even dimensions. We may be sacrificing the symmetries cited above, though. If the manifold is odd dimensional, however, we have to use the non-local, spectral boundary conditions. Atiyah and Bott [15 ] found out that in general there are topological obstructions to finding acceptable local boundary conditions. As described in $[15,16]$ the crucial point is defining an acceptable elliptic boundary problem for the signature 
operator whose index is the signature of the manifold. We call a complex elliptic if we can define an elliptic operator on it. The exterior algebra can be split into two distinct elliptic complexes. The first is the de Rham complex, which is related to the Euler characteristic. The second is the signature complex [17 ]. Although the de Rham complex admits local boundary conditions, it can be shown that for the signature complex, as well as for the spin and the Dolbeaux complexes, there does not exist a local boundary condition so that this complex with the local boundary condition is elliptic [18 ]. In the first order case, like the Dirac operator, there is a natural pseudo-differential operator given by the projection on the generalized eigenvectors with eigenvalues with plus or minus real parts. This operator leads to a well posed boundary value problem for the signature complex ( as well as the spin and the Dolbeaux complexes.) This fact requires the use of the spectral boundary conditions, which are non-local.

In defining the index of the operator, the presence of a boundary necessitates extra terms in addition to the Euler number. For the case without a boundary, knowing only the curvature suffices to compute the index [17 ]. One calculates the integral over the whole manifold of the same characteristic classes. If the manifold has a boundary, then, we have to add a term obtained from the integration of the Chern-Simons form, which is written in terms of the connection, the curvature and the second fundamental form determined from the normal to the boundary. Another term that should be added is the $\eta$ invariant, which is calculated from the eigenvalues of the operator restricted to the boundary. We also add a term proportional to the number of zero eigenvalue solutions of this operator. The index is equal to the sum of these four terms.

Here we study the Dirac equation in the background of the helicoid metric in five and four dimensions in consecutive sections. We do not attempt to calculate the index for our Dirac operator. Our only aim is to make a sense out of this incomplete metric by restricting the domain and using the appropriate boundary conditions for this problem.

\section{Solutions of Dirac Equations}

The relevance of the helicoid- catenoid metric, as a simplest minimal surface in Euclidean space resulting in a gravitational instanton [1], for certain cosmological models is given in a recent preprint [21]. One can transform from the helicoid to the catenoid metric by a simple transformation [2]. The importance of five dimensional spaces is also stressed in the same paper. The helicoid metric is also mentioned among the metrics related to $\mathbf{M}$ theory by Gibbons et al [22]. This metric is also studied by Valent in [23,24,25]. These are other reasons why we try to give a meaning to the solutions of Dirac equation in the background of the Nutku helicoid solution both in four and five dimensions. Here we briefly introduce the metric and write formally the Dirac equation in the background of this metric.

The Nutku helicoid metric is written for the four dimensional Euclidean space. It is given as [2] 


$$
d s^{2}=\frac{1}{\sqrt{1+\frac{a^{2}}{r^{2}}}}\left[d r^{2}+\left(r^{2}+a^{2}\right) d \theta^{2}+\left(1+\frac{a^{2}}{r^{2}} \sin ^{2} \theta\right) d y^{2}-\frac{a^{2}}{r^{2}} \sin 2 \theta d y d z+\left(1+\frac{a^{2}}{r^{2}} \cos ^{2} \theta\right) d z^{2}\right]
$$

where $0<r<\infty, 0 \leq \theta \leq 2 \pi, y$ and $z$ are along the Killing directions and will be taken to be periodic coordinates on a 2 -torus. This is an example of a multi-center metric. This metric reduces to the flat metric if we take $a=0$.

If we make the following transformation $r=\operatorname{asinh} x$, the metric is written as

$$
d s_{4}^{2}=\frac{a^{2}}{2} \sinh 2 x\left(d x^{2}+d \theta^{2}\right)+\frac{2}{\sinh 2 x}\left[\left(\sinh ^{2} x+\sin ^{2} \theta\right) d y^{2}-\sin 2 \theta d y d z+\left(\sinh ^{2} x+\cos ^{2} \theta\right) d z^{2}\right] .
$$

We use the NP formalism [26,27] in four Euclidean dimensions $[28,29,30]$. The details of how the tetrad is chosen, how the $\gamma^{\mu}$ matrices are formed for this explicit case are given in [5 ].

We use the Dirac operator $i \gamma^{\mu} \nabla_{\mu}$ where

$$
\nabla_{\mu}=\partial_{\mu}-\Gamma_{\mu}
$$

The $\gamma$ matrices satisfy

$$
\left\{\gamma^{\mu}, \gamma^{\nu}\right\}=2 g^{\mu \nu}
$$

The spin connection is written as $\Gamma_{\mu}=\frac{1}{4} \gamma_{; \mu}^{\nu} \gamma_{\nu}$.

We can trivially extend this structure to five dimensions The addition of the Euclidean "time component" to the previous metric gives:

$$
d s^{2}=d t^{2}+d s_{4}^{2}
$$

Going to five dimensions does not increase the number of equations we have to solve, since in both four and five dimensions we can use four component Dirac spinors. We can not interpret the fifth dimension as time, since this will bring problems with causality. If we use non-local boundary conditions, these conditions are set for all times. Abrikosov has devised a new method [31] which cures the causality problem. We could not, however, generalize this method to our case, since our little Dirac equation still couples three components. We, therefore, in both even and odd dimensional cases, study the solution with the Euclidean metric. We include both cases to compare and contrast the similarities and differences of these closely related cases.

\section{Solutions in five dimensions}

We start by studying the solutions to the Dirac equation in the background of the Nutku helicoid metric in five dimensions. Our motivation is to see whether one can find a domain where solutions in the background of this singular metric can be defined. We will try to show that one can obtain a satisfactory solution to this problem. 
We write the system in the form $L \psi=\Lambda \psi$ and try to obtain the solutions for the different components. Our aim is to write the upper components in terms of the lower components. We will impose the boundary conditions on the upper components in terms of derivatives of the lower components below.

The equations read

$$
\begin{aligned}
& \frac{\sqrt{2}}{a \sqrt{\sinh 2 x}}\left\{\left(\partial_{x}+i \partial_{\theta}\right) \Psi_{3}+a\left[\cos (\theta+i x) \partial_{y}+\sin (\theta+i x) \partial_{z}\right] \Psi_{4}-\frac{a \sqrt{\sinh 2 x}}{\sqrt{2}} \partial_{t} \Psi_{1}\right\}=\Lambda \Psi_{1}, \quad[6 a] \\
& \frac{\sqrt{2}}{a \sqrt{\sinh 2 x}}\left\{\left(\partial_{x}-i \partial_{\theta}\right) \Psi_{4}-a\left[\cos (\theta-i x) \partial_{y}+\sin (\theta-i x) \partial_{z}\right] \Psi_{3}-\frac{a \sqrt{\sinh 2 x}}{\sqrt{2}} \partial_{t} \Psi_{2}\right\}=\Lambda \Psi_{2}, \quad[6 b] \\
& \frac{\sqrt{2}}{a \sqrt{\sinh 2 x}}\left\{\left(\partial_{x}-i \partial_{\theta}+\operatorname{coth} 2 x\right) \Psi_{1}-a\left[\cos (\theta+i x) \partial_{y}+\sin (\theta+i x) \partial_{z}\right] \Psi_{2}+\frac{a \sqrt{\sinh 2 x}}{\sqrt{2}} \partial_{t} \Psi_{3}\right\}=\Lambda \Psi_{3}, \quad[6 c] \\
& \frac{\sqrt{2}}{a \sqrt{\sinh 2 x}}\left\{\left(\partial_{x}+i \partial_{\theta}+\operatorname{coth} 2 x\right) \Psi_{2}+a\left[\cos (\theta-i x) \partial_{y}+\sin (\theta-i x) \partial_{z}\right] \Psi_{1}+\frac{a \sqrt{\sinh 2 x}}{\sqrt{2}} \partial_{t} \Psi_{4}\right\}=\Lambda \Psi_{4} . \quad[6 d]
\end{aligned}
$$

These are coupled equations involving three different components. The usual method to obtain solutions at this stage is the separation of variables method. Three of the variables, $t, y, z$, define the Killing directions. This is exploited by writing the solution as a product of exponentials in $y, z, t$ times a function of $x$ and $\theta$.

$$
\Psi_{i}=e^{i\left(k_{t} t+k_{y} y+k_{z} z\right)} \Psi_{i}(x, \theta) .
$$

We take $k_{y}=k \cos (\phi), k_{z}=k \sin (\phi)$, since then we can absorb the variable $\phi$ in functions of the remaining variables $x$ and $\theta$.

We note that these four equations are not similar in form. The transformation $\Psi_{1,2}=\frac{1}{\sqrt{\sinh 2 x}} f_{1,2}$ is used for the upper components. This transformation eliminates the $\operatorname{coth} 2 x$ terms in the third and the fourth equations. With this transformation, the equations read

$$
\begin{gathered}
\left\{\left(\partial_{x}+i \partial_{\theta}\right) \Psi_{3}+i a k[\cos (\theta-\phi+i x)] \Psi_{4}-i a k_{t} f_{1}\right\}=\Lambda \frac{a}{\sqrt{2}} f_{1}, \\
\left\{\left(\partial_{x}-i \partial_{\theta}\right) \Psi_{4}-i a k[\cos (\theta-\phi-i x)] \Psi_{3}-i a k_{t} f_{2}\right\}=\Lambda \frac{a}{\sqrt{2}} f_{2}, \\
\left(-\partial_{x}+i \partial_{\theta}\right) f_{1}+i a k[\cos (\theta-\phi+i x)] f_{2}+i a k_{t} \Psi_{3}=-\Lambda \frac{a \sinh 2 x}{\sqrt{2}} \Psi_{3}, \\
\left(-\partial_{x}-i \partial_{\theta}\right) f_{2}-i a k[\cos (\theta-\phi-i x)] f_{1}+i a k_{t} \Psi_{4}=-\Lambda \frac{a \sinh 2 x}{\sqrt{2}} \Psi_{4} .
\end{gathered}
$$

We solve our equations in terms of $f_{1,2}$ and substitute these expressions in equations, given above. This substitution gives us second order, but uncoupled equations for the lower components.

$$
\left(\partial_{x x}+\partial_{\theta \theta}+\frac{a^{2}}{2}\left[k^{2}(-\cos [2(\theta-\phi)]-\cosh 2 x)-\left(k_{t}^{2}+\Lambda^{2}\right) \sinh 2 x\right]\right) \Psi_{3,4}=0 .
$$


We can separate this equation into two ordinary differential equations by the ansatz $\Psi_{3,4}=R(x) S(\theta-\phi)$. Separation of equation(9) gives us two ordinary differential equations. The equation for $S$ reads

$$
\partial_{\Theta \Theta} S(\Theta)-\left(\frac{a^{2}}{2} k^{2} \cos (2 \Theta)-n\right) S(\Theta)=0,
$$

where $(\theta-\phi)=\Theta$. This equation is of the Mathieu type and the solution can be written immediately.

$$
S(\theta)=C_{1} S e\left(n, \frac{a^{2} k^{2}}{4}, \theta-\phi\right)+C_{2} S o\left(n, \frac{a^{2} k^{2}}{4}, \theta-\phi\right)
$$

The solutions should be periodic in the angular variable $\Theta$. This fact forces $n$, the separation constant, to take discrete values. It is known that the angular Mathieu functions satisfy an orthogonality relation such that functions with different $n$ values are perpendicular to each other. Here, we integrate the angular variable from zero to $2 \pi$. One can fix the normalization constant according to the chosen normalization, whether it is according to the McLachlan or the Morse-Stratton convention [32 ].

The equation for $R(x)$ reads

$$
\left\{\partial_{x x}-\left[\frac{a^{2}}{2}\left(k^{2} \cosh 2 x+\left(k_{t}^{2}+\Lambda^{2}\right) \sinh 2 x\right]+n\right\} R(x)=0 .\right.
$$

whose solution can be reduced to the form

$$
R(x)=D_{1} S e\left(n, A_{6}, i(x+b)\right)+D_{2} S o\left(n, A_{6}, i(x+b)\right) .
$$

Here $C_{1}, C_{2}, D_{1}, D_{2}$ are arbitrary constants. The other constants will be defined below as we explain how one can reduce our initial equation to give these solutions. The solutions for the lower components $\psi_{3}, \psi_{4}$ are given in terms of sums over $n$ and integrals over $k, \phi, k_{t}$ we used in the separation ansatz. We think it is amusing to explain how these solutions are obtained. To get this solutions one has to go through several steps.

In reference books the solution of eq. (13) is not listed as a Mathieu function. In fact, we find that the solution of this equation is expressed in terms of double confluent Heun functions [33 ]. We denote these functions as $H_{D}$ in our expressions.

$$
\begin{gathered}
R(x)=C_{1} H_{D}\left(0, \frac{a^{2} k^{2}}{2}-n, a^{2}\left(\Lambda^{2}+k_{t}^{2}\right), \frac{a^{2} k^{2}}{2}+n, \tanh x\right) \\
+C_{2} H_{D}\left(0, \frac{a^{2} k^{2}}{2}-n, a^{2}\left(\Lambda^{2}+k_{t}^{2}\right), \frac{a^{2} k^{2}}{2}+n, \tanh x\right) \\
\times \int \frac{-d x}{H_{D}\left(0, \frac{a^{2} k^{2}}{2}-n, a^{2}\left(\Lambda^{2}+k_{t}^{2}\right), \frac{a^{2} k^{2}}{2}+n, \tanh x\right)^{2}}
\end{gathered}
$$

Normally one takes the first function and discards the second solution.

Reading through literature [34 ] we suspect that the solution we found can be expressed in terms of Mathieu functions after performing proper transformations. We can show that we can indeed express the result in 
terms of Mathieu functions by using a simpler independent variable than the one given in eq. (14) after performing few transformations. We define

$$
\begin{gathered}
A_{1}=\frac{-a^{2}\left(k_{t}^{2}+\Lambda^{2}\right)}{2}, \\
A_{2}=-n \\
A_{3}=-\frac{a^{2} k^{2}}{2},
\end{gathered}
$$

and use the transformation

$$
z=e^{-2 x}
$$

Then the differential operator in eq. (12) is expressed as

$$
\left(4 z^{2} \partial_{z z}+4 z \partial_{z}+\left(\frac{A_{3}-A_{1}}{2}\right) z+A_{5}+\left(\frac{A_{3}+A_{1}}{2}\right) \frac{1}{z}\right) f=0 .
$$

This equation is still of the double confluent Heun form, since it still has two irregular singularities at zero and infinity. We define $A_{4}=\left(\frac{A_{3}-A_{1}}{2}\right)$ and $A_{5}=\left(\frac{A_{3}+A_{1}}{2}\right)$. If we take

$$
\sqrt{\frac{A_{4}}{A_{5}}} u=z
$$

and

$$
w=\frac{1}{2}\left(u+\frac{1}{u}\right)
$$

and set $A_{6}=\sqrt{A_{4} A_{5}}$ we get,

$$
\left(\left(w^{2}-1\right) \partial_{w w}+w \partial_{w}+\left(\frac{A_{6}}{2} w+\frac{A_{2}}{4}\right)\right) f=0 .
$$

With the new transformations, we have traded the irregular singularity at zero by two regular singularities at plus and minus one. This is the same singularity structure of the Mathieu equation. The solution of this equation is indeed expressible in terms of Mathieu functions. It is given as:

$$
R(w)=S e\left(n, A_{6}, \arccos \sqrt{\frac{w+1}{2}}\right)+S o\left(n, A_{6}, \arccos \sqrt{\frac{w+1}{2}}\right),
$$

where $A_{6}=\frac{a^{2}}{4}\left[k^{4}-\left(k_{t}^{2}+\Lambda^{2}\right)^{2}\right]^{1 / 2}$. Going back through the transformations we made, it is not hard to express $\arccos \sqrt{\frac{w+1}{2}}$ in terms of our original variable $x$ up to a constant, $i(x+b)$. We simply write

$$
\frac{w+1}{2}=\frac{e^{-2 x}\left(\frac{e^{2 x}+\sqrt{k^{2}-k_{t}^{2}-\Lambda^{2}}}{\left(k^{2}+k_{t}^{2}+\Lambda^{2}\right.}\right)^{2}}{4 \sqrt{\frac{k^{2}-k_{t}^{2}-\Lambda^{2}}{\left(k^{2}+k_{t}^{2}+\Lambda^{2}\right)}}}=\cosh ^{2}(x+b) .
$$

Here

$$
e^{-2 b}=\sqrt{\frac{k^{2}-k_{t}^{2}-\Lambda^{2}}{k^{2}+k_{t}^{2}+\Lambda^{2}}}
$$


Taking the arccos of this expression gives the result given in eq. (13).

After these transformations, we see that the solution of the lower components of the Dirac equation can be expressed in terms of functions that are regular at zero. We can not say this for the upper components, though. They are expressed in terms of these solutions and their derivatives divided by a function, which blows up at zero. Even if we take the so called odd Mathieu functions, which can be expanded in terms of hyperbolic sine functions, their derivatives will be hyperbolic cosine functions. To obtain the upper components we have to divide them by $\sqrt{\sinh 2 x}$. Then these functions will still blow at the origin.

A finite scalar product can be defined around the origin for these solutions in the form

$$
\int \Psi_{i}^{*} \Psi_{i} \sqrt{g} d \tau, i=1-4
$$

in a finite domain, including the origin. Here $d \tau$ is the volume element in our five dimensional space. Repeated indices are not summed over. $\sqrt{g}$ is the square root of the determinant of the metric, necessary to get an invariant volume element. The zero of the invariant measure cancels the singularity of the wave functions at the origin.

We find, however, that the solutions are not normalizable as $x$ goes to infinity. Furthermore, our metric has curvature singularities at the origin. Although, using our new measure, we can make all four components normalizable at the origin, we still define our solutions for the domain $0<x<F$. We integrate $x$ variable in the domain up to the point $x \leq F$, where $F$ is the point the function starts to diverge. Since our radial solution is multiplied by the angular solution and the exponential function to make up the total solution of the Dirac equation, the orthogonality of the angular solutions for different values of the discrete $n$ makes our solutions orthogonal to each other. By dividing by the appropriate factors, we can normalize them.

We note that the domain where the solutions are normalizable is restricted. These differential equations do not have a meaning unless we define the boundary conditions the solutions obey at the boundary of our domain. Since our system exists in an odd dimensional manifold with a boundary, we have to study this equation using spectral boundary conditions $[9,10]$.

The method used in applying these boundary conditions requires first studying the little Dirac equation, the tangential operator of the Dirac operator restricted to the boundary, where the variable $x$ takes a fixed value $x_{0}$. We have to take $x_{0}$ greater than zero since the second fundamental form defined by our choice of the normal to the boundary diverges at $x_{0}=0[18]$.

For this purpose we write the equations in the form given in equations (8), i.e. after $\Psi_{1,2}$ are transformed to $f_{1,2}$.

$$
\begin{aligned}
& \frac{\sqrt{2}}{a}\left\{i \partial_{\theta} \Psi_{3}+i a k \cos \left(\theta-\phi+i x_{0}\right) \Psi_{4}-\frac{i a k_{t}}{\sqrt{2}} f_{1}\right\}=\lambda f_{1}, \\
& \frac{\sqrt{2}}{a}\left\{-i \partial_{\theta} \Psi_{4}-i a k \cos \left(\theta-\phi-i x_{0}\right) \Psi_{3}-\frac{i a k_{t}}{\sqrt{2}} f_{2}\right\}=\lambda f_{2}, \\
& \frac{\sqrt{2}}{a}\left\{-i \partial_{\theta} f_{1}-i a k \cos \left(\theta-\phi+i x_{0}\right) f_{2}+\frac{i a k_{t}}{\sqrt{2}} \Psi_{3}\right\}=\lambda \Psi_{3},
\end{aligned}
$$




$$
\frac{\sqrt{2}}{a}\left\{i \partial_{\theta} f_{2}+i a k \cos \left(\theta-\phi-i x_{0}\right) f_{1}+\frac{i a k_{t}}{\sqrt{2}} \Psi_{4}\right\}=\lambda \Psi_{4} .
$$

Here $\lambda$ is the eigenvalue of the little Dirac equation.

We could not obtain analytical solutions of these equations in terms of known functions. We could not even write uncoupled equations in the second order. One needs to go to fourth order in derivatives to be able to write equations that involves a single unknown function.

At this point we follow closely our references $[6,7,8,31]$. We formally expand our solutions at the boundary, fixed by two values of $x_{0}$ in terms of eigenfunctions of the little Dirac equations with both positive and negative eigenvalues $\lambda$. For the lower components we have

$$
\Psi_{3,4}^{\Lambda}\left(\Theta, x_{0}\right)=\sum_{\lambda} h_{\lambda}\left(\Theta, x_{0}\right)
$$

for fixed values of $k_{t}, k_{y}, k_{z}$. We set

$$
\left.\Psi_{3,4}^{\Lambda}\left(\Theta, x_{0}\right)\right|_{\partial B}=\sum_{\lambda>0} h_{\lambda}\left(\Theta, x_{0}\right)
$$

The negative $\lambda$ eigenvectors are all set to be zero at the boundary.

Then, we solve $f_{1,2}$ in terms of $\Psi_{3,4}$ using the equations (8a, 8b) and fix the $x$ values to $x_{0}$ on the boundary. Note that since $x=x_{0}$, the derivative with respect to $x$ is evaluated at this point as well as the terms without the $x$ derivative. We can, in general, use the expansion given for $\Psi_{3,4}$ on the boundary, in terms of its eigenfunctions.

$$
\Psi_{3,4}^{\Lambda}\left(\Theta, x_{0}\right)=\sum_{\lambda} h_{\lambda, 3,4}\left(\Theta, x_{0}\right) .
$$

This sum is over all values of $\lambda$. In fixing the values of $f_{1,2}$ in terms of $\Psi_{3,4}$ on the boundary, we use only part of the expansion of $\Psi_{3,4}^{\Lambda}(\Theta, x)$ where

$$
\left.\Psi_{3,4}^{\Lambda}(\Theta, x)\right|_{\partial B}=\sum_{\lambda<0} h_{\lambda, 3,4}\left(x_{0}, \Theta\right)
$$

In other words, we write $\left.\Psi_{1,2}^{\Lambda}(\Theta, x)\right|_{\partial B}$ using the expressions obtained from $f_{1,2}$ in terms of the negative $\lambda$ values of $\left.\Psi_{3,4}^{\Lambda}(\Theta, x)\right|_{\partial B}$. These boundary conditions are non local, but are shown to be the only consistent ones for odd dimensional Euclidean spaces by Atiyah-Patodi-Singer.

\section{Solutions in four dimensions}

Here we repeat the calculations given above after setting $k_{t}$ to zero. Our aim is to show that the five dimensional case, studied above, is not essentially different from the four dimensional case. Since both solutions can be expressed in terms of Mathieu functions we can compare the four and five dimensional cases. These equations with $\Lambda=0$ were studied in ref. [5 ]. The solutions obtained in this reference for the four dimensional case can be summed to give the Green's function similar to the calculation done in ref. [2 
]. This is not possible for the five dimensional solution of ref. [5 ]. For the solutions with $\Lambda \neq 0$ both in five and four dimensions this property does not exist. The radial and the angular equations have different constants which does not allow to obtain the Green's function of the equation by using summation formulae of Mathieu functions [35].

Our solutions exist only in a finite domain for the variable $x$, hence we have to use the appropriate boundary conditions at this point. Using the orthogonality of the angular Mathieu functions for different values of the discrete parameter $n$, we can show the orthogonality of these solutions.

At this point, although we can use local boundary conditions in this case, we choose to use the spectral boundary conditions of Atiyah-Patodi-Singer to conserve chirality and charge conjugation. We are keen not to break chirality by hand, since in the standard model this symmetry is expected to break spontaneously, resulting in confinement. There are cases, however, to describe the correct physics, where one may want to break this symmetry to force the system to one of the broken phases. Using chirality breaking boundary conditions may be one way to achieve this task. This is discussed in detail in reference [36]. In this work, we do not want to break the chiral symmetry of the model. We, therefore, adhere to the spectral boundary conditions.

We write the system in the form $L \psi=\Lambda \psi$ where $\psi$ is a four component spinor, and try to obtain the solutions for the different components. Then our equations are similar to the ones given in the equations (6). The only difference is taking $k_{f}$ equal to zero. We see that three components are still coupled in our equations.

The method of solution is exactly like the one used in the previous section. To get our solutions we use the separation of variables method. We write the solution as a product of exponentials in $y, z$ times a function of $x$ and $\theta$.

$$
\Psi_{i}=e^{i\left(k_{y} y+k_{z} z\right)} \Psi_{i}(x, \theta)
$$

The same transformations are used as those in the five dimensional case to reduce $\Psi_{1,2}$ to $f_{1,2}$. We solve our equations in terms of $f_{1,2}$ and substitute these expressions in equations, given above. We end up with second order, but uncoupled equations for the lower components.

$$
\left(\partial_{x x}+\partial_{\theta \theta}-\frac{a^{2}}{2}\left[k^{2}(\cos 2(\theta-\phi)+\cosh 2 x)+\Lambda^{2} \sinh 2 x\right]\right) \Psi_{3,4}=0
$$

We can separate this equation into two ordinary differential equations by the ansatz $\Psi_{3,4}=R(x) S(\theta)$. For $S(\Theta)$ we get an equation of the Mathieu type and the solution can be written immediately.

$$
S(\theta)=C_{1} \operatorname{Se}\left[n, \frac{a^{2} k^{2}}{4}, \theta-\phi\right]+C_{2} S o\left(n, \frac{a^{2} k^{2}}{4}, \theta-\phi\right) .
$$

Solution for $R(x)$ can be reduced to

$$
R(x)=D_{1} S o\left(n, B, i\left(x+b^{\prime}\right)\right)+D_{2} S e\left(n, B, i\left(x+b^{\prime}\right)\right),
$$


Here $C_{1}, C_{2}, D_{1}, D_{2}$ are arbitrary constants. $B=\frac{\sqrt{k^{4}-\Lambda^{4}}}{2} . b^{\prime}$ is defined as in eq.(25) with $k_{t}=0$ Just note that $n$ is the separation constant which has to take discrete values to get a periodic solutions for the angular Mathieu equation $S(\Theta)$. The solutions for the lower components $\Psi_{3}, \Psi_{4}$ are given in terms of sums over $n$ and integrals over $k, \phi$ we used in the separation ansatz.

We find that we have the upper solutions, $\Psi_{1,2}$ are divergent at the origin, whereas the lower ones are finite. Both of our solutions diverge at infinity. For the same reasons as given for the five dimensional case, we have to limit the domain of our solutions at two finite values of $\mathrm{x}$.

To impose these boundary conditions we need to write the little Dirac equation, the Dirac equation restricted to the boundary, where the variable $x$ takes a fixed value $x_{0}$. We choose to write the equations in the form,

$$
\begin{aligned}
& \frac{\sqrt{2}}{a}\left\{i \partial_{\theta} \Psi_{3}+i k a \cos \left(\theta-\phi+i x_{0}\right) \Psi_{4}\right\}=\lambda f_{1}, \\
& \frac{\sqrt{2}}{a}\left\{-i \partial_{\theta} \Psi_{4}-i a k \cos \left(\theta-\phi-i x_{0}\right) \Psi_{3}\right\}=\lambda f_{2}, \\
& \frac{\sqrt{2}}{a}\left\{\left(-i \partial_{\theta}\right) f_{1}-i a k \cos \left(\theta-\phi+i x_{0}\right) f_{2}\right\}=\lambda \Psi_{3}, \\
& \frac{\sqrt{2}}{a}\left\{\left(i \partial_{\theta}\right) f_{2}+i a k \cos \left(\theta-\phi-i x_{0}\right) f_{1}\right\}=\lambda \Psi_{4} .
\end{aligned}
$$

Here $\lambda$ is the eigenvalue of the little Dirac equation.

We could not obtain analytical solutions of these equations in terms of known functions. This is the same result as in the five dimensional case. For us it seems very curious being able to solve similar system of partial differential equations, but not even being able to decouple them when this system reduces to ordinary differential equations on the boundary. One possible explanation is that $\theta-\phi \pm i x$ act as $z$ and $\bar{z}$ of complex variables. Sometimes it is easier to find functions of this pair as solutions is easier than a function of a single real variable. Actually for the full Dirac equation, Sucu and Ünal [3] find solutions in a closed form. Same technique, however, does not seem to work when $x=x_{0}$.

From equations (36) we see that we can take $\Psi_{4}$ as the complex conjugate of $\Psi_{3}$ and $f_{2}$ as the complex conjugate of $f_{1}$. We take $f_{1}=p_{1}+i q_{1}$ and $\Psi_{3}=p_{3}+i q_{3}$. We end up with coupled differential equations for these functions. One notes, however, that separating the real and imaginary parts of each solution, we get

$$
\begin{gathered}
\partial_{\Theta \Theta} p_{1}+a k\left[-\sin \Theta \cosh x_{0}-\left(\sin ^{2} \Theta \sinh ^{2} x_{0}+\cos ^{2} \Theta \cosh ^{2} x_{0}\right)\right] p_{1}-a k \cos \Theta \sinh x_{0} q_{1}=\lambda^{2} p_{1}, \\
\partial_{\Theta \Theta} q_{1}+a k\left[\sin \Theta \cosh x_{0}-\left(\sin ^{2} \Theta \sinh ^{2} x_{0}+\cos ^{2} \Theta \cosh ^{2} x_{0}\right)\right] q_{1}-a k \cos \Theta \sinh x_{0} p_{1}=\lambda^{2} q_{1} .
\end{gathered}
$$

We see that the eigenvalue of the little Dirac equation comes only quadratically, showing a symmetry for its positive and negative values. This will make the $\eta$ invariant, defined as

$$
\eta=\lim _{s \rightarrow 0} \sum_{\lambda_{i}} \operatorname{sign}\left(\lambda_{i}\right)\left|\lambda_{i}\right|^{-s}
$$


which is needed for an index calculation, zero. We also see a symmetry between $p_{1}$ and $q_{1}$, namely $p_{1}(\Theta)=$ $q_{1}(-\Theta)$. If we, instead, eliminate $p_{1}, q_{1}$ and write our equations for $p_{3}, q_{3}$, we get exactly the same equations. We expand our solutions at the boundary, fixed by two values of $x_{0}$ in terms of eigenfunctions of the little Dirac equations with both positive and negative eigenvalues $\lambda$.

$$
\Psi_{i}^{\Lambda}\left(\Theta, x_{0}\right)=\sum_{\lambda} g_{i, \lambda}\left(\Theta, x_{0}\right)
$$

for fixed values of $k_{y}, k_{z}$. We set

$$
\left.\Psi_{3,4}^{\Lambda}(\Theta, x)\right|_{\partial B}=\sum_{\lambda>0} g_{\lambda, 3,4}\left(\Theta, x_{0}\right) .
$$

The negative $\lambda$ eigenvectors are all set to be zero at the boundary.

The boundary conditions on the upper components are imposed exactly in the same manner as explained in the five dimensional case, namely we solve for $f_{1,2}$ in terms of $\Psi_{3,4}$ using the equations

$$
\begin{aligned}
& \frac{\sqrt{2}}{a}\left\{\left(\partial_{x}+i \partial_{\theta}\right) \Psi_{3}+a[\cos (\theta-\phi+i x)] \Psi_{4}\right\}=\Lambda f_{1}, \\
& \frac{\sqrt{2}}{a}\left\{\left(\partial_{x}-i \partial_{\theta}\right) \Psi_{4}-a[\cos (\theta-\phi-i x)] \Psi_{3}\right\}=\Lambda f_{2},
\end{aligned}
$$

and fix the $x$ values to $x_{0}$ on the boundary.

We can in general use the expansion given for $\Psi_{3,4}$ on the boundary, in terms of its eigenfunctions, eqn. (40). This sum is over all values of $\lambda$. In fixing the values of $f_{1,2}$ in terms of $\Psi_{3,4}$ on the boundary, we use only the part where $\lambda<0$. These boundary conditions are non local, but they respect self adjointness and conserve $\gamma^{5}$ and charge conjugation symmetry.

\section{Conclusion}

Here we tried to give solutions of the Dirac equation in five dimensions for the Nutku helicoid metric in a bounded region. We found out that they can be reduced to Mathieu functions, which is also the case in four dimensions. We imposed formally [8,31] the non-local spectral boundary conditions of Atiyah-Patodi and Singer $[9,10,6,7]$ on these solutions, which are the only correct boundary conditions in odd dimensions. Our main goal in this paper is to define the solutions of the Dirac equation in the background of a incomplete metric, namely the Nutku helicoid solution consistently.

A related work would be to calculate the index of the Dirac operator in four dimensions in this background. From our solutions we can calculate both the bulk and the surface term easily. From the form of the little Dirac equation, we see that the $\eta$ invariant is zero. We could not obtain analytical solutions of the little Dirac equation, though. We give examples of the numerical solutions we found of the zero eigenvalue equations of the little Dirac equation in Figure 1. These pictures do not correspond to any of the functions we encounter in the literature $[32,33,35,37]$. Therefore, we know that the zero mode solutions of the little Dirac equation 
exist. We, however, do not know their analytical expression, hence their number. As a result, we could not calculate the index in this paper.

Acknowledgement: We thank Profs. John Roe, Yavuz Nutku, Ayşe Bilge and Neşe Özdemir for correspondence, for discussions and scientific assistance throughout this work. The work of M.H. is also supported by TUBA, the Academy of Sciences of Turkey. This work is also supported by TUBITAK, the Scientific and Technological Council of Turkey.

\section{References}

1. Nutku Y 1996 Phys.Rev. Lett. 774702

2. Aliev A N, Hortaçsu M, Kalaycı J and Nutku Y 1999 Class. Quantum Grav. 16631

3. Sucu Y and Ünal N 2004 Class. Quant. Grav. 211443

4. Villalba V M 2005 J.Phys.: Conf. Ser. 24136

5. Birkandan T and Hortaçsu M 2007 J.Phys. A 40 1105, e-Print Archive: gr-qc/0607108 and corrigendum to be published

6. Hortaçsu M, Rothe K D, Schroer B 1980 Nucl. Phys. B171 530

7. Hortaçsu M 1983 Lettere al Nuovo Cim. 36109

8. Abrikosov, jr. A A, Wipf A 2007 J. Phys. A: Math. Theor. 405163

9. Atiyah M F, Patodi V K and Singer I M 1975 Math. Proc. Camb.Phil. Soc. 7743

10. Atiyah M F, Patodi V K and Singer I M 1975 Math. Proc. Camb.Phil. Soc. 77405

11. Peeters K and Waldron A 1999 JHEP 9902:024; e-Print Archive: hep-th/9901016

12. Roe J 1988 J. Differential Geom. 2787

13. Roe J 1988 J. Differential Geom. 27115

14. Falomir H 1997 e-Print Archive: physics/9705013

15. Atiyah M F and Bott R 1964, Index Theorem for Manifolds with Boundary in : Differential Analysis (Bombay Colloquium) Oxford Univ. Press

16. Gilkey P B, Kirsten K and J.H. Park J. H. 2005 J.Phys.A 388103, e-Print Archive: math-ph/0406028

17. Gilkey P B 1984 Invariance Theory, the Heat Equation and the Atiyah-Singer Index Theorem Publish or Perish Inc., Delaware, USA.

18. Eguchi T, Gilkey P.B and Hanson A 1980, Physics Reports C66 213

19. Gilkey P B 1984 Invariance Theory, the Heat Equation and the Atiyah-Singer Index Theorem Publish or Perish Inc., Delaware, USA. p.248 Lemma 4.1.6

20. Esposito G Dirac Operators and Spectral Geometry Cambridge Lect.Notes Phys.12:1-209,1998 Cambridge Univ. Press, Cambridge, England 
21. Popescu A S 2007, "Dimension Embedded in Unified Symmetry", e-Print Archive: gr-qc/0704.2670

22. Gibbons G W and Rychenkova P 2000 J. Geometry and Phys. 32311

23. Valent G and Ben Yahia H 2007 Class. and Quant. Grav. 24255

24. Valent G 2004 Commun. Math. Phys. 244571

25. Valent G 2005 Int. J. Mod. Phys. A 202500

26. Newman E T and Penrose R 1962 J. Math. Phys. 3566

27. Newman E T and Penrose R 1962 J. Math. Phys. 4998

28. Goldblatt E 1994 Gen.Rel. Grav. 26979

29. Goldblatt E 1994 J. Math. Phys. 353029

30. Aliev A N and Nutku Y 1999 Class. Quantum Grav. 161892

31. Abrikosov jr. A A 2006 J.Phys.A 396109 e-Print Archive: hep-th/0512311

32. Gutiérrez Vega J C 2003 "Theory and Numerical Analysis of Mathieu Functions", Tecnológico de Monterrey, México report and the references given in this paper.

33. Ronveaux A (ed) 1995 Heun's Differential Equations (Oxford: Oxford University Press)

34. Schmidt D and Wolf G in: Ronveaux A (ed) 1995 Heun's Differential Equations (Oxford: Oxford University Press)

35. Morse P M and Feshbach H 1953 Methods of Theoretical Physics (McGraw Hill) p.1421

36. Wipf A and Duerr S 1995 Nucl. Phys.B 443202

37. Slavyanov S Yu, in :Ronveaux A (ed) 1995 Heun's Differential Equations (Oxford: Oxford University Press). p.95 


\section{FIGURE CAPTIONS}

Figure 1: Numerical solution for zero mode solutions of $p_{1}$ and $q_{1}$ in four dimensions for $x_{0}=0.005, a=$ $1, k=1$. 


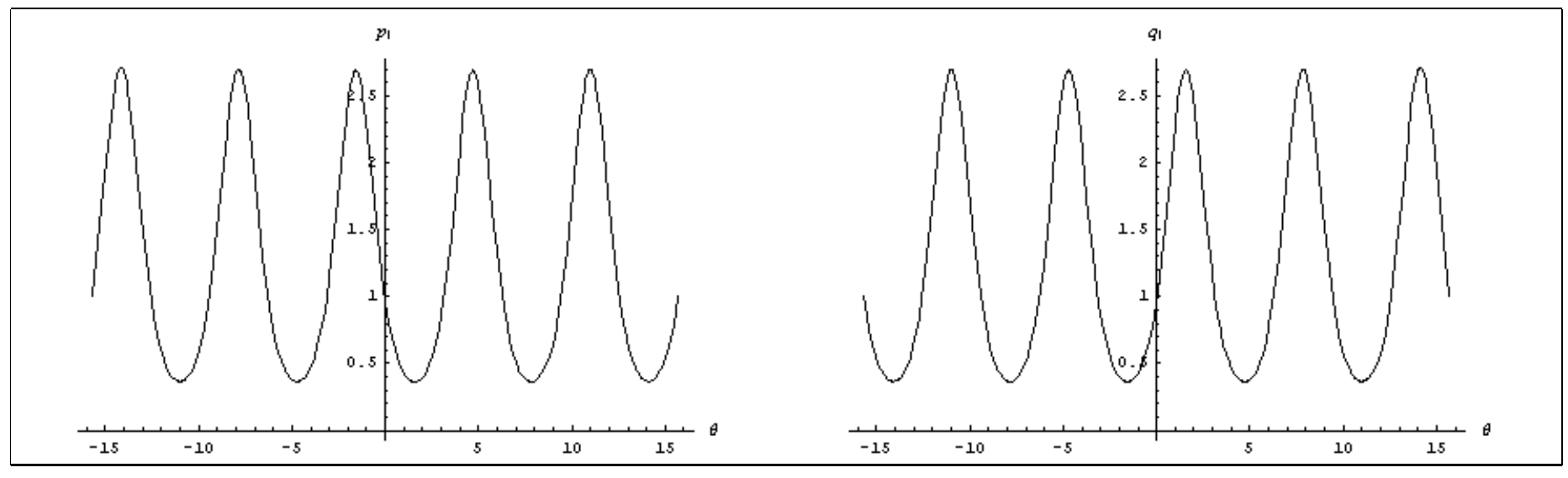

Figure 1 